\title{
Calcul en fiabilité d'un quai sur pieux à partir de données d'instrumentation
}

H. YÁÑEZ-GODOY

OXAND SA

49, av. Franklin-Roosevelt

77210 Avon, France humberto.yanez@oxand.com

\section{F. SCHOEFS}

A. NOUY

Université de Nantes

Institut de recherche en génie civil

et mécanique (GeM)

UMR 6183 CNRS

franck.schoefs@univ-nantes.fr
Les résultats de l'instrumentation d'un quai sur pieux ont permis d'établir une méthodologie de mise à jour des calculs de fiabilité par instrumentation des structures. Cette approche repose sur la prise en compte de manière probabiliste des phénomènes naturels et mécaniques (marée, vent, poussée du remblai) et de certaines conditions aux limites (dont l'interaction sol-tirant-plaque d'ancrage).

\section{Reliability assessment of a harbour structure from structural monitoring}

In this paper the instrumentation of a pile-supported wharf is the support to establish the updating of a reliability assessment by structural instrumentation. This approach is based on the taking into account of the natural and mechanical phenomena (tide, wind and embankment loading) and of certain boundary conditions (for example the soil-rod-anchoring plate interaction) in a probabilistic way. 


\section{Introduction}

Le calcul en service d'ouvrages complexes où le sol joue un rôle déterminant reste encore un défi. Il est pourtant souvent nécessaire dès qu'il s'agit de prolonger la durée de service de certaines structures. Lorsqu'un remblai est réalisé, les conditions de mise en œuvre présentent un aléa important et rendent difficile, voire impossible toute modélisation ultérieure du comportement réel. L'instrumentation est une piste explorée depuis une dizaine d'années pour différents types de structures dont une revue est disponible dans Verdure (2004). D'un coût acceptable $(0,8$ à 1,3\% du projet), celle-ci fournit en effet de précieuses informations. Des analyses inverses sont alors nécessaires si l'on souhaite passer de l'analyse des données à la modélisation.

Cet article présente une application à partir d'une structure pilote de type quai sur pieux. On introduit tout d'abord la problématique du calcul en fiabilité de structures existantes. L'ouvrage et son instrumentation sont présentés. Les résultats sont commentés. La phase de modélisation, des séries temporelles et de la mécanique de l'ouvrage est ensuite proposée en vue d'une identification des variables. L'article se conclut sur l'identification des variables et le calcul de fiabilité du quai.

\section{2}

\section{Problématique du calcul en fiabilité de structures existantes}

Le calcul de fiabilité implique la construction d'un critère de défaillance classiquement appelé fonction d'état limite ou marge de sécurité dans des cas linéaires. L'état limite inclut non seulement les caractéristiques structurales qui assurent la portance des divers chargements (pour une période prédéfinie), mais aussi les critères de confort.

On considère un critère d'état limite de type performantiel : $\mathrm{G}=\mathrm{u}_{\max }-\mathrm{u}_{\mathrm{c}^{\prime}}$ où $\mathrm{u}_{\mathrm{c}}$ est le déplacement critique qui traduit l'exigence du gestionnaire en terme de déplacement de la structure et $u_{\max }$ est le déplacement maximal le long de l'ouvrage. Le gestionnaire du Port Autonome de Nantes - Saint-Nazaire (PANSN) suggère un déplacement maximal le long du quai de 0,01 m. En effet, le déplacement de la plate-forme du quai engendre un déplacement du sol, un décompactage, puis des tassements irréversibles derrière la plate-forme à la jonction entre cette dernière et le remblai (Fig. 1). Cette situation rend l'ouvrage quasiment inexploitable. Les déplacements sont calculés avec un modèle 2-D (Yáñez-Godoy, 2008) qui prend en compte une variable aléatoire caractérisant le comportement spatial du quai et les sollicitations du vent (Yáñez-Godoy et al., 2008a). La probabilité de défaillance $P_{f}$ est alors calculée. Elle est définie par : $\mathrm{P}_{\mathrm{f}}=\mathrm{P}\left(\mathrm{u}_{\max }>0,01 \mathrm{~m}\right)$.

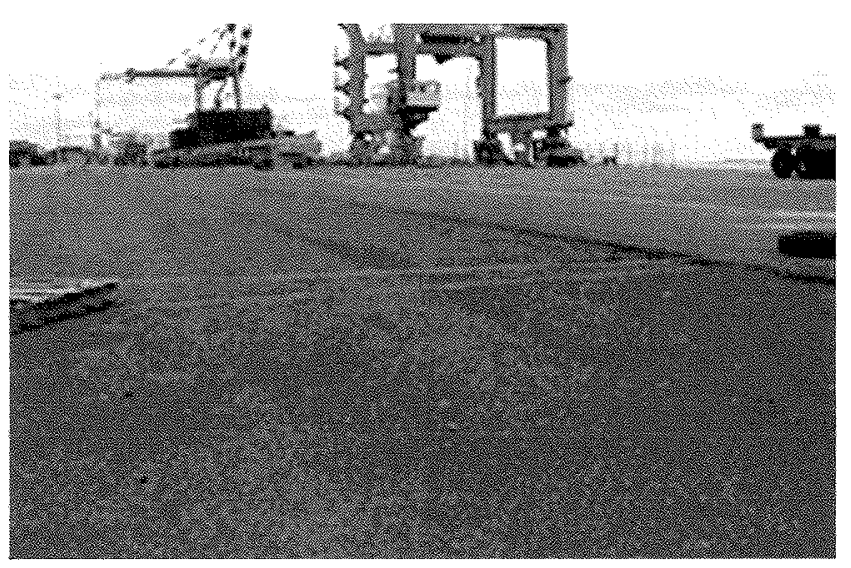

Hc. 1 Tassements de la zone aval du quai TMDC4, Montoir.

Settlings in the downstream zone of TMDC-4 wharf.

\section{3}

\section{L'ouvrage et son instrumentation}

\section{3}

\section{Présentation de l'ouvrage}

L'ouvrage étudié concerne l'extension du terminal à bois de Cheviré, poste 4 (référencé quai C-4 par la suite). Le quai C-4 est situé en aval du pont de Cheviré, à proximité de Nantes, sur la rive gauche de la Loire. Il s'agit d'un quai sur pieux de $180 \mathrm{~m}$ de longueur et $34,50 \mathrm{~m}$ de largeur, prévu pour recevoir des navires de longueur maximale de $225 \mathrm{~m}$ et d'un tirant d'eau de $9,10 \mathrm{~m}$. La figure 2 décrit les éléments principaux du quai C-4.

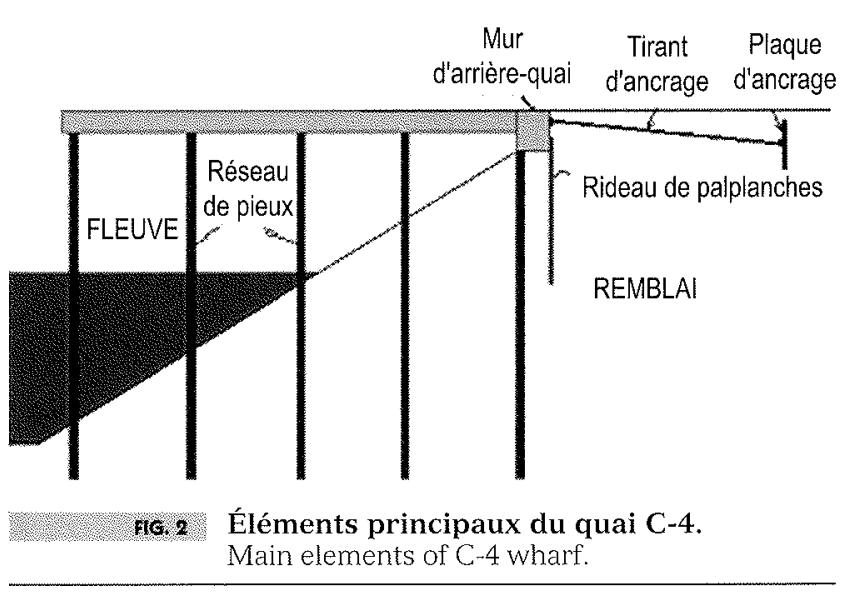

On considère ici uniquement le comportement quasi statique de ce quai. Les situations de chargement comprennent : (1) le chargement vertical provenant du poids propre de la structure, des grues et du stockage ; (2) le chargement horizontal venant de la poussée du remblai sur le mur d'arrière-quai (actions dues au remblayage et aux variations de la marée), l'accostage et l'amarrage des navires et des actions du vent sur les grues. Les hypothèses principales, généralement admises, proviennent d'avis d'experts et d'études d'incertitudes réalisées pendant la préparation de l'Eurocode 7 (Magnan, 2006). On se concentre ici sur les charge- 
ments pour lesquels les risques et les incertitudes sont les plus importants : chargements horizontaux et particulièrement celui agissant de la rive vers le fleuve.

\section{2}

\section{Instrumentation structurale}

L'objectif étant d'analyser le comportement du quai sous chargement horizontal, on a choisi d'instrumenter les tirants d'ancrage, éléments sensibles du quai et non accessibles après le chantier, et de suivre des grandeurs comme le déplacement du quai, le niveau de la marée et de la nappe dans le remblai. Le quai étant peu exploité durant la prise des mesures, et aucune relation entre la présence d'un navire (sans tempête) et le niveau d'effort n'ayant pu être mise à jour, seuls les chargements de remblai ont été analysés.

Douze tirants d'ancrage ont été instrumentés. Régulièrement répartis sur la longueur de l'ouvrage, ils ont été repérés et nommés par une lettre " $T$ » et leur abscisse longitudinal $x$ en mètre, afin de suivre l'effort normal (Fig. 3). L'originalité de cette instrumentation se trouve dans la volonté de disposer d'une forte proportion de tirants instrumentés (30\%) permettant de dégager les variations spatiales et de réaliser des modélisations probabilistes s'appuyant sur un traitement statistique. Le choix d'une instrumentation située derrière l'arrière-quai permet d'éviter le traitement du frottement latéral sur les tirants, identifié comme phénomène important par Verdure (2004) et constaté aussi sur le quai du port de Calais (Delattre et Mespoulhé, 1999), sur le quai d'Osaka au Havre (Delattre et al., 1999), sur le quai Port 2000 (Marten, 2005) et sur le quai Altenwerder (Gatterman et al., 2001).

Par ailleurs, des mesures de hauteur de nappe d'eau dans le remblai ont été effectuées à l'aide de trois piézomètres implantés derrière le mur d'arrière-quai et reliés au poste d'acquisition (de type Campbell Scientific « CR10X ») qui centralise l'ensemble des données. Un marégraphe a mesuré le niveau réel de la marée toutes les cinq minutes. Le marégraphe du PANSN était situé à $1 \mathrm{~km}$ en aval du pont de Cheviré. Les données ont été rapportées au zéro des CM.

\section{4}

\section{Résultats de l'instrumentation}

La modélisation du comportement de tirants passifs dans des remblais reste un sujet d'investigation en géotechnique. Certains calculs par éléments finis ont permis de représenter en partie le comportement observé, mais la question de l'ajustement des paramètres reste délicate (Marten, 2005). Avec l'instrumentation, on cherche donc à caractériser un comportement global du complexe (c sol-tirant-plaque d'ancrage ) (STPA).

On encadre les analyses des mesures d'efforts dans les tirants d'ancrage du quai en ciblant les deux types de variations qui caractérisent ces efforts :

- variations temporelles : des variations à moyen terme, où se pose la question des niveaux d'efforts au cours d'un même mois (une période de révolution de la lune) et des variations à court terme, où on s'est intéressé à l'amplitude des efforts durant une marée au cours d'une demi-journée ;

- variations spatiales, d'un tirant à l'autre.

Parmi les résultats importants (Yáñez-Godoy, 2008), on a constaté une forte dispersion dans l'espace et le temps :

- des profils d'efforts repris par les tirants le long du quai (Fig. 4) ;

- de la sensibilité des tirants à la marée.

Le premier point renvoie aux conditions initiales au cours du chantier. En effet, le constat du passage répété d'engins d'approvisionnement au niveau des tirants T0 à T65 au cours du chantier a pu entraîner un compactage plus important du remblai sur la partie amont du quai.

Le second point est directement lié à la raideur du complexe STPA. Yáñez-Godoy et al. (2008b) ont montré, qu'en présence de forts coefficients de marée (supérieurs à 90), le comportement de chaque tirant pouvait être représenté par un coefficient de sensibilité à la marée, défini comme le ratio entre la variation des efforts au cours d'une marée et la variation de hauteur d'eau dans le remblai correspondant. En situation de tempête, on se trouve en général dans la situation de

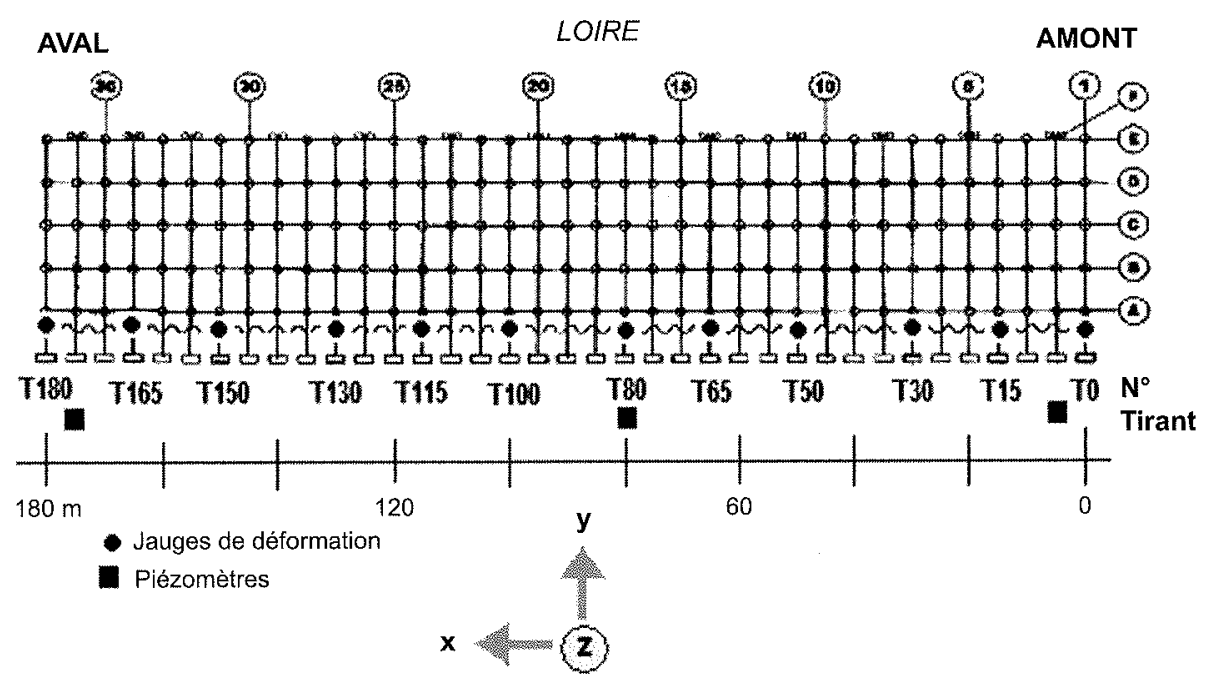

FG. 3 Schéma de la répartition de l'instrumentation le long du quai C-4. Instrumentation implanted along the C-4 wharf. 


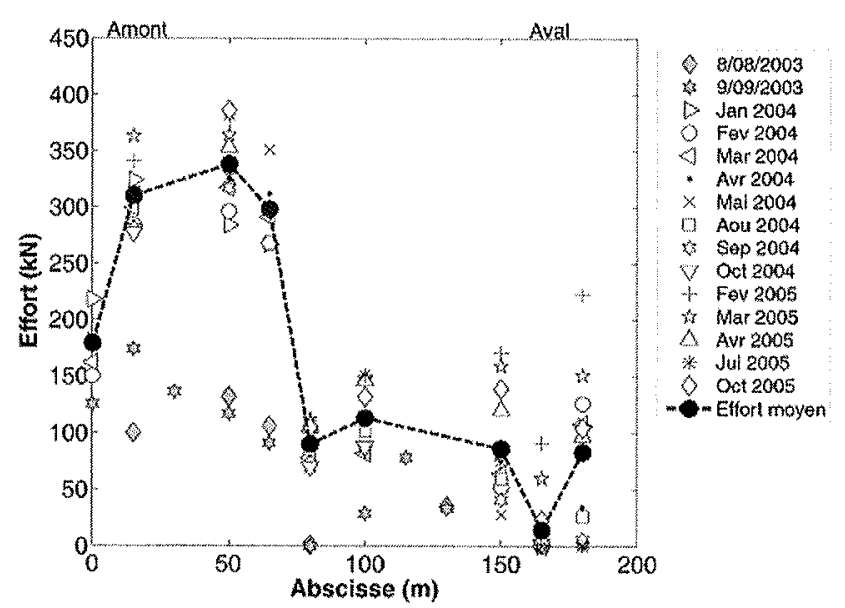

196. 4 Variabilité de l'effort à moyen terme le long du quai C-4.

Medium-term evolution of the monthly average measured load in the tie-rods along the $\mathrm{C}-4$ wharf.

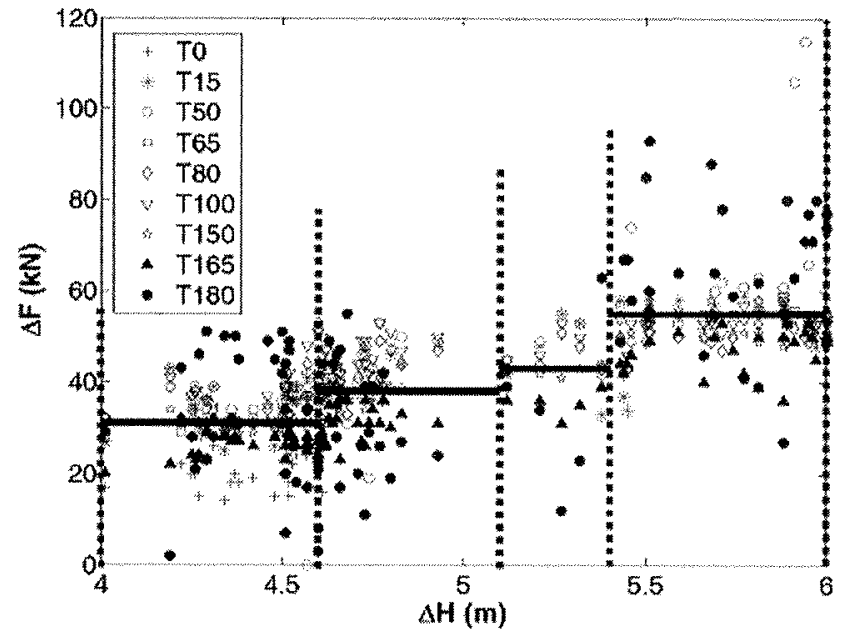

F6. Variation de l'effort $\Delta F$ en fonction $d u$ niveau d'eau de la Loire $\Delta \mathrm{H}$.

Variation of the measured normal load in the tie-rods according to water level variation of the river Loire during falling tides of high coefficient.

par l'abscisse $x$ et en temps $t, F(x, t, \theta)$. Les capteurs enregistrent des réalisations de trajectoires de ce processus : $\mathrm{F}\left(\mathrm{x}_{\mathrm{i}}, \mathrm{t}_{\mathrm{j}}, \theta_{\mathrm{k}}\right)$, où $\theta_{\mathrm{k}}$ représente l'aléa généré par l'événement $\mathrm{k}$. On observe alors un effort total dans les tirants caractérisé par:

$$
F(x, t, \theta)=F_{r}(x, t)+F_{m}(x, t, \theta)
$$

où $F_{r}(x, t)$ est la composante permanente due au chargement du remblai seul $F_{R}$ et $F_{m}(x, t, \theta)=k(x, t, \theta) . u(x)$ représente la composante due au chargement de la marée, c'est-à-dire, au déplacement du quai u(x) à partir de la position d'équilibre sous la poussée de la marée sur le remblai et en fonction de la raideur $\mathrm{k}(\mathrm{x}, \mathrm{t}, \boldsymbol{\theta})$ du système STPA.

Toutes les mesures ont été obtenues sur une période de non exploitation du quai. On considère donc un découplage à ce niveau. Ce choix évite de résoudre un problème d'optimisation à un grand nombre de degrés de liberté (chargement extérieur, paramètres du modèle mécano-probabiliste, etc.).

D'abord, on suppose que le processus stochasticue de chargement des tirants est stationnaire et ergodique en temps. En particulier à un instant $t_{j}$ on ne dispose que d'une réalisation $\mathrm{F}\left(\mathrm{x}_{\mathrm{i}}, \mathrm{t}_{\mathrm{j}}, \theta_{\mathrm{k}}\right)$ du processus. Par la suite et par abus d'écriture, on fait référence à ce processus de cette manière : $F(x, \theta)$. Cela implique que chaque réalisation de cette variable provient d'une mesure à un temps donné.

La génération du champ $F(x, \theta)$ par une seule variable $\mathrm{k}(\boldsymbol{\theta})$ permet de réduire la complexité pour se concentrer sur un développement robuste de la méthode d'identification des caractéristiques de $\mathrm{k}$. Le comportement au cours des marées de fort coefficient conduit à l'identification de la raideur équivalente $\mathrm{k} d u$ complexe STPA pour le quai (voir $\$ 5.2$ ).

En l'absence de tout autre chargement, le remblai génère un pré-chargement $F_{r}$ dans les tirants (éq. 1). La détermination du chargement déterministe du remblai $F_{R}$ est nécessaire en raison de la méconnaissance du chargement de la pression du sol et de l'impossibilité actuelle de prédire ce comportement avec des modèles numériques. En particulier, il a été montré (Verdure, 2004 ; Verdure et al., 2005) que les calculs d'ana- 
lyse limite ne fournissent pas une borne supérieure aux efforts mesurés. Les méthodes aux éléments finis impliquent, quant à elles, le calage d'un grand nombre de paramètres a priori inconnus ou non mesurables directement dans une telle situation (Marten, 2005). Il paraît difficile dans ces conditions de proposer un modèle robuste de chargement aléatoire de remblai. On reporte alors tout aléa et incertitude sur les paramètres du modèle mécano-probabiliste.

Le chargement déterministe dû à la poussée des terres $F_{R}$ est cherché en le supposant uniforme sur tout le quai, représenté par des efforts ponctuels en chaque point d'ancrage des tirants sur l'arrière quai et calculé par une analyse inverse en utilisant l'algorithme du simplex présenté dans Lagarias et al. (1998). Un modèle mécanique de type poutre de Timoshenko libre (modèle 2-D) permet de réduire considérablement les temps de calcul. Ses paramètres ont été identifiés à partir d'un modèle complet 3-D du quai (Yáñez-Godoy, 2008). Le problème d'optimisation correspondant se résout par la méthode des moindres carrés comme suit :

$$
\begin{gathered}
F_{\mathrm{R}}=\underset{\mathrm{F}_{\mathrm{R}}}{\operatorname{argmin}}\left(\lambda\left(\mathrm{F}_{\mathrm{R}}\right)\right) \\
\lambda\left(F_{R}\right)=\left(\sum_{i=1}^{n}\left(F_{r, \text { mes }}\left(X_{i}\right)-F_{r}^{c}\left(X_{i}, F_{\mathrm{R}}, k_{d}\right)\right)^{2}\right)
\end{gathered}
$$

où $\lambda\left(F_{R}\right)$ est la fonction de performance; on repère par $x_{1} l^{\prime}$ abscisse des points où on dispose de mesures; $\mathrm{F}_{r}^{\mathrm{c}}\left(\mathrm{x}_{\mathrm{i}}, \mathrm{F}_{\mathrm{R}}, \mathrm{k}_{\mathrm{d}}\right)$ est l'effort dans le tirant d'ancrage d'abscisse $x_{i}$ résultant d'un calcul avec le modèle 2-D en appliquant un chargement du remblai $F_{R}$ et avec une raideur $\mathrm{k}_{\mathrm{d}}$, étant prise à une valeur nominale de $61,9 \mathrm{MN} / \mathrm{m}$, correspondant à la raideur d'un tirant seul parfaitement encastré dans la plaque d'ancrage sans considérer l'interaction avec le sol.

Les résultats du chargement déterministe du remblai sont présentés sur la figure 6 . Les points 1 et 8 se trouvent ( décalés ) par rapport aux points voisins; un défaut de compaction du remblai et de pose du mécanisme de liaison du tirant pourraient expliquer ces irrégularités (Yáñez-Godoy et al., 2008b).

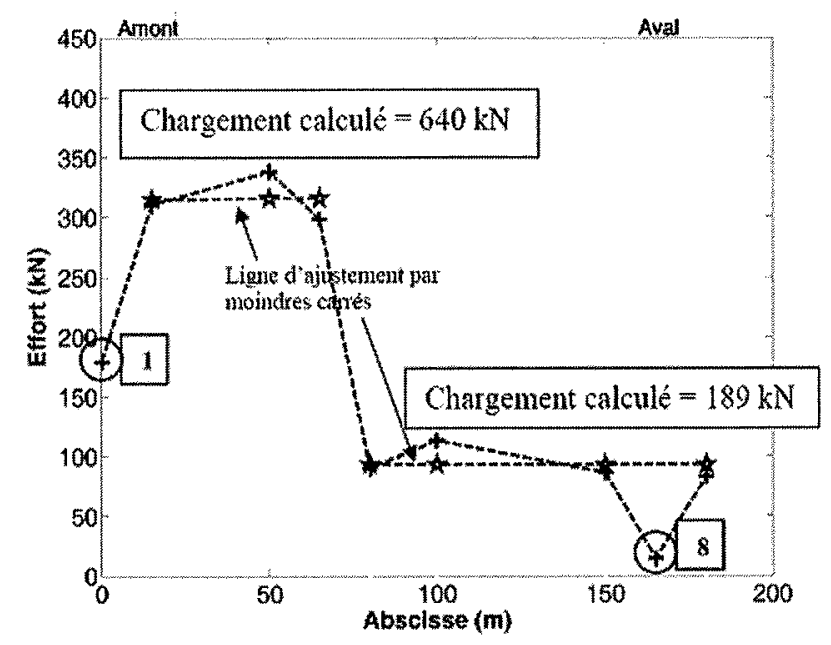

16. 6. Calcul de la poussée du remblai $F_{R}$. Computed embankment loading.

\section{9}

\section{Identification des caractéristiques de la variable aléatoire $k$}

Afin d'identifier la variable $\mathrm{k}(\boldsymbol{\theta} \mid \Delta \mathrm{H})$, qui représente la raideur du complexe STPA, on considère trois étapes (Yáñez-Godoy, 2008) :

a) estimation, pendant une marée descendante, de l'évolution du chargement déterministe du remblai, pour chaque intervalle $\mathrm{I}_{\Delta \mathrm{H}^{\prime}}$ appelé chargement de marée $\Delta \mathrm{F}_{\mathrm{CM}}\left(\mathrm{I}_{\Delta \mathrm{H}}\right)$ (Fig. 5);

b) calcul des événements $\mathrm{k}\left(\theta \quad \mathrm{II}_{\Delta \mathrm{H}}\right)$ connaissant $\Delta \mathrm{F}_{\mathrm{CM}}\left(\mathrm{I}_{\Delta \mathrm{H}}\right)$;

c) identification de la distribution $\operatorname{de~} \mathrm{k}\left(\boldsymbol{\theta} \mid \mathrm{I}_{\Delta \mathrm{H}}\right)$ en utilisant l'identification sur le chaos polynomial (YáñezGodoy et al., 2008a).

L'étape (a) est effectuée comme pour l'obtention de $F_{\mathrm{R}}$. Le problème d'optimisation correspondant se résout comme suit:

$$
\begin{gathered}
\Delta F_{C M}\left(I_{\Delta H}\right)=\underset{\Delta F_{C M}\left(I_{\Delta H}\right)}{\operatorname{argmin}}\left(\lambda\left(\Delta F_{C M}\left(I_{\Delta H}\right)\right)\right) \\
\lambda\left(\Delta F_{C M}\left(I_{\Delta H}\right)\right)=\sum_{i=1}^{n}\left(\Delta F^{c}\left(X_{i}, \Delta F_{C M}\left(I_{\Delta H}\right), k_{d}\right)-\Delta F\left(I_{\Delta H}\right)\right)^{2}
\end{gathered}
$$

où $\lambda\left(\Delta \mathrm{F}_{\mathrm{CM}}\left(\mathrm{I}_{\Delta \mathrm{H}}\right)\right)$ est la fonction de performance ; $\Delta \mathrm{Fc}^{c}\left(\mathrm{x}_{\mathrm{i}}, \Delta \mathrm{F}_{\mathrm{CM}}\left(\mathrm{I}_{\Delta \mathrm{H}}\right), \mathrm{k}_{\mathrm{d}}\right)$, la variation de l'effort dans le tirant d'ancrage $d^{\prime}$ abscisse $x_{i}$ résultant d'un calcul avec le modèle 2-D, en appliquant un chargement cle marée $\Delta \mathrm{F}_{\mathrm{CM}}\left(\mathrm{I}_{\Delta \mathrm{H}}\right)$ et avec $\mathrm{k}_{\mathrm{cl}}=61,9 \mathrm{MN} / \mathrm{m} ; \Delta \mathrm{F}\left(\mathrm{I}_{\Delta \mathrm{H}}\right)$ pour chaque $\mathrm{I}_{\Delta H}$ est obtenu à partir de la figure 5 et est égale à : 32 , 38,43 et $55 \mathrm{kN}$. Les valeurs obtenues pour $\Delta \mathrm{F}_{\mathrm{CM}}\left(\mathrm{I}_{\Delta \mathrm{H}}\right)$ sont les suivantes : $64,77,87$ et $111 \mathrm{kN}$.

Pour l'étape $(b)$, chaque événement $\mathrm{k}\left(\theta_{1} \backslash \Delta \mathrm{H}\right)$ constitue une solution du problème d'optimisation :

$$
\begin{gathered}
k\left(\theta_{l} \mid I_{\Delta H}\right)=\underset{k}{\arg \min }\left(\lambda\left(k\left(\theta_{l} \mid I_{\Delta H}\right)\right)\right) \\
\lambda\left(k\left(\theta_{l} \mid I_{\Delta H}\right)\right)=\sum_{i=1}^{n}\left(\Delta F\left(X_{i}, \theta_{l} \mid I_{\Delta H}\right)-\Delta F^{c}\left(X_{i}, \Delta F_{C M}\left(I_{\Delta H}\right), k\right)\right)^{2}
\end{gathered}
$$

où $\lambda\left(\mathrm{k}\left(\theta_{I} \mid I_{\Delta \mathrm{H}}\right)\right)$ est la fonction de performance ; $\Delta \mathrm{FC}^{\mathrm{C}}\left(\mathrm{X}_{\mathrm{i}}, \Delta \mathrm{F}_{\mathrm{CM}}\left(\mathrm{I}_{\Delta \mathrm{H}}\right), \mathrm{k}\right)$, la variation de l'effort dans un tirant $\mathrm{d}^{\prime}$ abscisse $\mathrm{X}_{\mathrm{i}}$ résultant d'un calcul avec le modèle 2-D, en appliquant un chargement de marée $\Delta \mathrm{F}_{\mathrm{CM}}\left(\mathrm{I}_{\Delta \mathrm{H}}\right)$, déterminée dans l'étape (a), et avec une raideur $\mathrm{k}$. La série d'événements pour $\mathrm{k}(\theta \mid \Delta \mathrm{H})$ est donc déduite de la solution de $\mathrm{m}$ problèmes inverses, où $\mathrm{m}$ représente la taille des échantillons de $\Delta \mathrm{F}\left(\mathrm{x}_{\mathrm{i}^{\prime}}, \theta \mathrm{I}_{\Delta \mathrm{H}}\right)$. Ce problème se résout en utilisant l'algorithme du simplex.

Pour l'étape (c), on a choisi d'utiliser l'estimation du maximum de vraisemblance pour identifier la décomposition du chaos polynomial (voir définition en Annexe) (Desceliers et al., 2007). L'objectif est d'identifier les coefficients $k_{i}$ de la décomposition unidimensionnelle du chaos polynomial à partir de $N$ échantillons notés $\left\{\mathrm{x}\left(\theta_{\mathrm{i}}\right)\right\}_{\mathrm{j}=1}^{\mathrm{N}}$. Cette décomposition s'écrit:

$$
k(\theta)=k(\xi(\theta))=\sum_{i=0}^{p} k_{i} h_{j}(\xi(\theta))
$$

où $\mathrm{p}$ est l'ordre de la décomposition; $\xi(\theta)$, le germe Gaussien (chaos unidimensionnel), i.e. une variable normalisée et $h_{i}$, le polynôme d'Hermite de degré i. En utilisant la méthode du maximum de vraisemblance, les coefficients $\mathrm{k}_{\mathrm{i}}$ sont solution du problème d'optimisation suivant : 


$$
\kappa=\underset{\kappa}{\operatorname{argmax}} \mathrm{L}(\kappa)
$$

où $\kappa=\left[\mathrm{k}_{0}, \ldots, \mathrm{k}_{\mathrm{p}}\right] \in \mathbb{R}^{p}$ est le vecteur de coefficients de dimension $(p+1)$ et $L$, la fonction de vraisemblance :

$$
L(\kappa)=\prod_{j=1}^{N} p_{k}\left(k\left(\theta_{j}\right) ; \kappa\right)
$$

où $\mathrm{N}$ représente la taille de l'échantillon de $\mathrm{k}(\boldsymbol{\theta})$ et $p_{k}(. ; \kappa)$ la fonction de densité de probabilité de la variable $\mathrm{k}(\dot{\theta})$ qui dépend de l'ensemble des coefficients du chaos polynomial. La fonction de vraisemblance de l'équation 7 prend d'abord des valeurs voisines de la précision numérique. Le problème (éq. 6) est alors modifié de la façon suivante:

$$
\kappa=\underset{\kappa}{\operatorname{argmin}}(-\log (\operatorname{L}(\kappa)))
$$

L'algorithme de résolution est décrit dans YáñezGodoy (2008) et Schoefs et al. (2007). La figure 7 présente les résultats des ajustements à la distribution de $\mathrm{k}$ pour un chaos polynomial d'ordre 1,2 et 3 . On observe que la distribution la mieux ajustée aux mesures est obtenue pour l'ordre 3. Les valeurs de la moyenne et de l'écart-type de $\mathrm{k}(\xi(\theta))$ sont respectivement : $61,2 \mathrm{MN} / \mathrm{m}$ et 9,1 MN/m. La valeur moyenne est autour de la valeur théorique $(61,9 \mathrm{MN} / \mathrm{m})$.

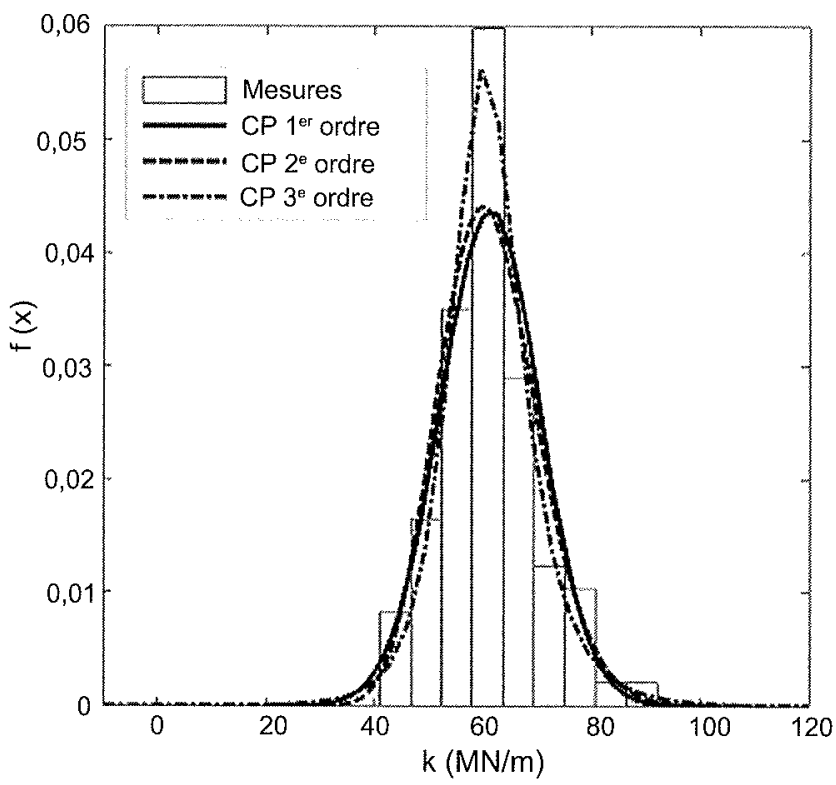

IG. 7 Résultats des ajustements à la distribution de $\mathbf{k}$ avec le chaos polynômial.

Distribution of the stiffness of the tie-rods for several degrees of polynomial chaos.

\section{Résultats du calcul de fiabilité}

Le calcul de fiabilité considère, d'un point de vue situation de calcul, la combinaison du chargement de tempête hivernale et le fort coefficient de marée. L'action du vent est modélisée par une surface de réponse agissant sur un portique et les forces résultantes Fv $(\theta)$ et FV $(\theta)$, corrélées entre elles, sont décomposées sur le chaošs polynomial (Yáñez-Godoy et al., 2008a).
Pour le calcul de la probabilité de défaillance, on recourt à des méthodes non intrusives (Berveiller, 2005) pour le calcul de la probabilité de dépassement du critère exigé. On s'intéresse aux déplacements maximaux $u_{\max }$ du quai, obtenus avec le modèle déterministe 2-D, où les chargements appliqués correspondent aux poussées du remblai à moyen terme, déterminés dans la section 5 , et aux efforts du vent au pied du portique. L'équation à résoudre est alors la suivante :

$$
\widetilde{u}_{\max }\left(k_{1}\left(\xi_{1}\right), F V_{2}\left(\xi_{2}\right)\right)=\sum_{j=0}^{P-1} u_{j} \Psi_{j}\left(\left\{\xi_{k}\right\}_{k=1}^{2}\right)
$$

Les coefficients $u_{j}$ sont obtenus par simple projection orthogonale sur la base multidimensionnelle de polynômes d'Hermite de gaussiennes centrées réduites $\psi_{j}\left(\left\{\xi_{k}\right\}_{k=1}^{2}\right\}$, où $\left\{\xi_{1}, \xi_{2}\right\}$ sont les variables aléatoires gaussiennes centrées réduites ayant servi à développer chacune des variables aléatoires d'entrée du système $\mathrm{k}$ et $\mathrm{Fv}(\theta)$; le cardinal $\mathrm{P}$ est $\mathrm{P}=\frac{(p+M) !}{M ! p !}$, où $\mathrm{M}=2$. L'algorithme de résolution de l'équation 9 est décrit dans Yáñez-Godoy (2008).

La figure 8 présente la distribution de la réponse du modèle de déplacement $u_{\max }$ du quai. En considérant $10 \mathrm{~mm}$ comme valeur de $u_{c^{\prime}}$ on obtient, à partir du postraitement de la figure 7 , une moyenne de $4,73 \mathrm{~mm}$ et un écart-type de 0,37 mm. La probabilité de défaillance $\left(\mathrm{P}_{\mathrm{f}}=\mathrm{P}\left(\mathrm{u}_{\max }>10\right)\right)$ obtenue est égale à $1,4 \cdot 10^{-7}$. L'exigence du gestionnaire de $10 \mathrm{~mm}$ est complètement remplie au sens des Eurocodes à l'ELS, donc à plus forte raison à l'ELU, en considérant l'effet du vent et les paramètres aléatoires. Grâce au surdimensionnement du quai, le fonctionnement en service n'est pas altéré par une situation de tempête. Les modifications ultérieures de cet ouvrage dues à son exploitation rendront primordiale cette analyse dans un cadre de maintenance et de requalification.

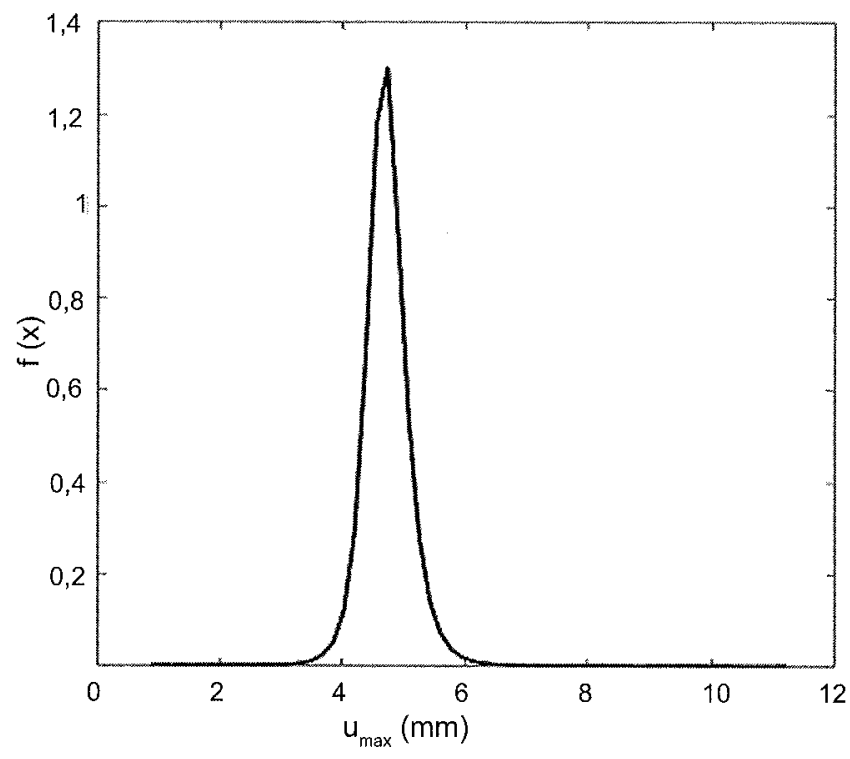

nG. 8 Résultat de la distribution de $u_{\max }$ par la méthode de projection.

Distribution of the computed maximal displacement. 


\section{Conclusion}

Dans le cas de structures complexes, le couplage de modèles mécaniques sophistiqués et de variables aléatoires rendant compte de la dispersion naturelle ou de l'incertitude des paramètres est très délicate, notamment parce qu'elle se heurte au problème de la caractérisation des variables (typologie des lois et paramètres ou premiers moments) et à celui du temps de calcul. Cet article a proposé, sur la base d'un modèle mécanique simple mais représentatif, une méthodologie pour identifier une variable aléatoire de type raideur caractérisant en situation de tempête le comportement du complexe « sol-tirant-plaque d'ancrage ». Un calcul de fiabilité a montré ensuite que la probabilité de défaillance est extrêmement faible.

\section{rexterciemartrs:}

Les auteurs tiennent à remercier le port autonome de Nantes - SaintNazaire pour son soutien technique Le premier auteur remercie le Consei National de Science et Technologie (CONACYT) du Mexicue pour avoir financé ces travaux de recherche.

\section{Annexe : Le chaos polynomial (Berveiller, 2005)}

Le chaos polynomial de dimension $M$ et d'ordre $p$ est défini comme l'ensemble des polynômes d'Hermite multidimensionnels en $\mathrm{M}$ variables aléatoires gaussiennes centrées réduites $\left\{\xi_{1}, \ldots, \xi_{M}\right\}$, dont le degré ne dépasse pas $\mathrm{p}$. Chacun de ces polynômes est complètement défini par une liste de $M$ entiers non négatifs $\left\{\alpha_{1}, \ldots, \alpha_{\mathrm{M}}\right\}$ comme suit :

$$
\Psi_{\alpha}=\prod_{i=1}^{M} h_{\alpha_{i}}\left(\xi_{i}\right), \alpha_{i} \geq 0
$$

où $h_{q}($.$) est le q-ième polynôme d'Hermite. On note$ aussi par $\partial_{\alpha}=\sum_{i=1}^{M} \alpha_{i}$ le degré de la liste $\alpha$. Le nombre de polynômes de $\mathrm{M}$ variables de degré inférieur ou égal à p est :

$$
P=\frac{(M+p) !}{M ! p !}
$$

\section{Bibliographie}

Berveiller M. - Éléments finis stochastiques : approches intrusive et non intrusive pour des analyses de fiabilité. Thèse Sciences de l'ingénieur, université Blaise-Pascal, Clermont II, laboratoire de Mécanique et Ingénieries, Clermont-Ferrand, 2005.

Delattre L., Mespoulhe L. - Étude expérimentale du comportement du quai en eau profonde du port de Calais. Rapport $n^{\circ}$ 65, Paris, France, LCPC, 1999, 193 p.

Delattre L., Duca V., Scherrer P., Rivière P. - Efforts d'ancrage du quai d'Osaka au port autonome du Havre. Proc. 12th Eur. Conf. on Soil Mech. and Geotech. Engin., 1999, p. 713-718.

Desceliers C., Soize C., Ghanem R. - Identification of chaos representations of elastic properties of random media using experimental vibration tests. Comp. Mech., 39 (6), 2007, p. 831-838.

Gattermann J., Bergs T., Rodatz W. - Modified instrumentation and results of stress and deformation monitoring at the new quay wall construction. Container Terminal Altenwerder, Port of Hamburg. Proc. 8th Int. Conf. on Struc. Safety and Reliab. (ICOSSAR'01), 2001.
Lagarias J.C., Reeds J.A., Wright M.H. Wright P.E. - Convergence properties of the Nelder-Mead simplex method in low dimensions. SIAM Journal of Optimization, 9(1), 1998, p. 112-147.

Magnan J.-P. - Eurocode 7 : calcul géotechnique. Tech. de l'ingénieur, traité Constr. C 240. France : Ed. T. I., Sciences et Techniques, 2006, p. C240 (1-8).

Marten S. - Étude expérimentale et méthodologique sur le comportement des écrans de soutènement. Thèse Sciences de l'ingénieur, École nationale de ponts et chaussées, LCPC, 2005, $300 \mathrm{p}$.

Schoefs F., Yáñez-Godoy H., Nouy A. - Identification of random material properties from monitoring of structures using stochastic chaos. Proc. 10th Int Conf on Applic of Stat and Prob. in Civil Engin. (ICASP10), 2007.

Verdure L. - Cadre statistique du suivi en service des ouvrages de génie civil: application à un quai sur pieux. Thèse Sciences de l'ingénieur, université de Nantes, Institut de recherche en génie civil et mécanique 2004
Verdure L.. Schoefs F., Casari P., YáñezGodoy $\mathrm{H}$. Uncertainty updating of an on-pile wharf after monitoring. Proc. 9th Int. Conf. on Struct. Safety and Reliab. (ICOSSAR), 2005, 1347-1354.

Yáñez-Godoy H., Schoefs F., Nouy A - Use of structural monitoring for updating reliability analysis : application on pile-supported wharves, Proc. of 4th Int ASRANet Colloquium, 25-27 June 2008, Athens, Greece, paper 74, 2008a, 10 p. proc. on CD-Rom.

Yáñez-Godoy H., Schoefs F., Casari P. - Statistical Analysis of the Effects of Building Conditions on the Initial Loadings of On-piles Quays. J. Struc. Health Monit., $7(3), 2008$ b, p. 245-263

Yáñez-Godoy H. - Mise à jour de variables aléatoires à partir des données d'instrumentations pour le calcul en fiabilité de structures portuaires. Thèse Sciences de l'ingénieur, université de Nantes, Institut de recherche en génie civil et mécanique, 2008. 